\title{
A Mobile, Multi-Camera Setup for 3D Full Body Imaging in Combination with Post-Mortem Computed Tomography Procedures
}

\author{
Sören KOTTNER ${ }^{1}$, Lars C. EBERT ${ }^{1}$, Garyfalia AMPANOZI ${ }^{1}$, \\ Marcel BRAUN ${ }^{2}$, Michael J. THALI ${ }^{1}$, Dominic GASCHO ${ }^{1}$ \\ ${ }^{1}$ Zurich Institute of Forensic Medicine, University of Zurich, Zurich, Switzerland; \\ ${ }^{2}$ Forensic Science Institute, Zurich, Switzerland
}

DOI: $10.15221 / 16.053 \quad$ http://dx.doi.org/10.15221/16.053

\begin{abstract}
Three dimensional (3D) models of deceased and injured people in combination with 3D scans of injury causing objects can assist forensic investigations in reconstructing event scenes. Medical imaging techniques, such as post-mortem computed tomography (PMCT) and post-mortem magnetic resonance imaging (PMMR), have been successfully applied to forensic investigations and can add beneficial value to standard autopsy examinations. These imaging modalities can be helpful for 3D reconstructions, especially when internal findings, such as bone fractures, organ damage and internal bleeding, are relevant for the investigation. However, none of these techniques can adequately visualize pattern injuries, such as boot prints and bite marks, or any type of blunt force trauma that forms distinct discolorations on the body's surface. This is why 3D surface imaging techniques have been introduced to the forensic community. Unfortunately, many commercially available optical scanning systems are cost intensive, time consuming and can only be applied before or after a CT scan has been performed. In this article, we present a mobile, multi-camera rig based on close-range photogrammetry that is inexpensive, fast in acquisition time and can be combined with automated CT scanning protocols. The multi-camera setup comprises seven digital single-lens reflex (DSLR) cameras that are mounted on a mobile frame. Each camera is equipped with a remote control that can trigger the shutter release of all cameras simultaneously. In combination with a medical CT scanner, image acquisition of the multi camera setup can be included into an automated CT scanning procedure. In our preliminary study, textured 3D models of one side of the body were created in less than 15 minutes. The photo acquisition time combined with the modified CT scanning protocols lasted 3:34 minutes whereas the subsequent computation of a textured 3D model based on a low resolution mesh lasted 10:55 minutes. The mobile, multi-camera setup can also be used manually in combination with examination couches, lifting carts and autopsy tables. Finally, the system is not limited to post-mortem investigations but can also be applied to living people and may be used in clinical settings.
\end{abstract}

Keywords: 3D body scanning, multi-camera setup, forensic imaging, 3D surface documentation

\section{Introduction}

Forensic imaging, especially three-dimensional (3D) imaging, has become a great help for forensic investigations. Three-dimensional data of injured and deceased people in combination with 3D scans or computer-aided design (CAD) models of injury causing objects can assist in reconstructing event scenes[1]-[4]. This reconstruction allows complicated three-dimensional relationships to be understood and visualized in a comprehensible way [1]-[8].

Medical imaging modalities, such as post-mortem computed tomography (PMCT) and post-mortem magnetic resonance imaging (PMMR), have been successfully applied to forensic investigations and have been proven to add beneficial value to standard autopsy examinations [9]-[15]. With respect to three-dimensional reconstructions of event scenes, these imaging techniques are especially helpful when internal findings, such as bone fractures, organ damage and internal bleeding, are relevant to the investigation [6], [7]. If, however, forensically relevant injuries form patterns on the body's surface due to discolorations (e.g., colour of haematomas or abrasions), neither of these imaging techniques can adequately visualize these findings. Injuries that form distinct discoloration patterns on the body's surface are called pattern injuries. These injuries can be the result of various types of blunt force trauma. Common examples are bite marks [4] and boot prints. To adequately document, measure and analyse pattern injuries, 3D surface imaging techniques have been introduced to the forensic community [16]-[18]. Unfortunately, most commercially available optical scanning systems are still cost intensive and can become time consuming when scanning a whole body. Furthermore, commercially available optical scanning systems can typically only be applied before or after a CT scan has been performed [8], [19]-[24]. 
Three-dimensional body scanning techniques have been used in a wide range of applications and are, of course, not only used in a forensic context [25]-[31]. The limitations, however, remain the same. Short scanning times are most practical to prevent motion artefacts due to, for example, changes in posture and respiration. This is why multi-camera setups have been used as body imaging systems [29], [32]-[36]. Chamber solutions with a cylindrical or spherical arrangement of cameras allow 360 degrees coverage of an object's surface [35], [37]. The overlapping 2D photographs that can be acquired when using a multi-camera setup can be used to reconstruct a textured $3 \mathrm{D}$ model based on, for example, a triangular mesh [38], [39].

In this technical note we introduce a mobile, multi-camera setup for 3D full body surface imaging. We present a semi-automatic usage in combination with post-mortem computed tomography procedures as well as a workflow for manual photo acquisition in combination with body carrying setups, such as examination couches, lifting carts and autopsy tables. The setup is based on a mobile frame and comprises seven digital cameras. Each camera is equipped with a radio controller that allows the simultaneous triggering of the shutter release of all cameras.

\section{Methods}

\subsection{The multi-camera setup}

The multi-camera rig comprises seven Canon EOS 100D (Canon Inc., Tokyo, Japan) digital single-lens reflex (DSLR) cameras. All camera bodies are equipped with a Canon EF lens with a fixed focal length of $50 \mathrm{~mm}$ and a wireless remote shutter control (Yongnou RF-603C II, YongNuo Photographic Equipment Co., Ltd, Shenzhen, China). The remote shutter release of all cameras is actuated simultaneously with a separate radio controller. Each camera is connected with a tripod ball head (G-10X, SIRUI Europe GmbH, Berlin, Germany or BALL 19P, NOVOFLEX Präzisionstechnik $\mathrm{GmbH}$, Memmingen, Germany) to a mobile right-angled frame. This frame is composed of two stands with a horizontal crosslink. Base plates are attached to the bottom end of the frame. Each base plate is equipped with swivel casters to ensure transportability of the system. At the top end of the frame, a mounting board allows adequate adjusting of the cameras to follow the arc of a circle. An additional laptop shelf is attached to the right stand of the frame. An overview of the multi-camera setup is provided in Figure 1.

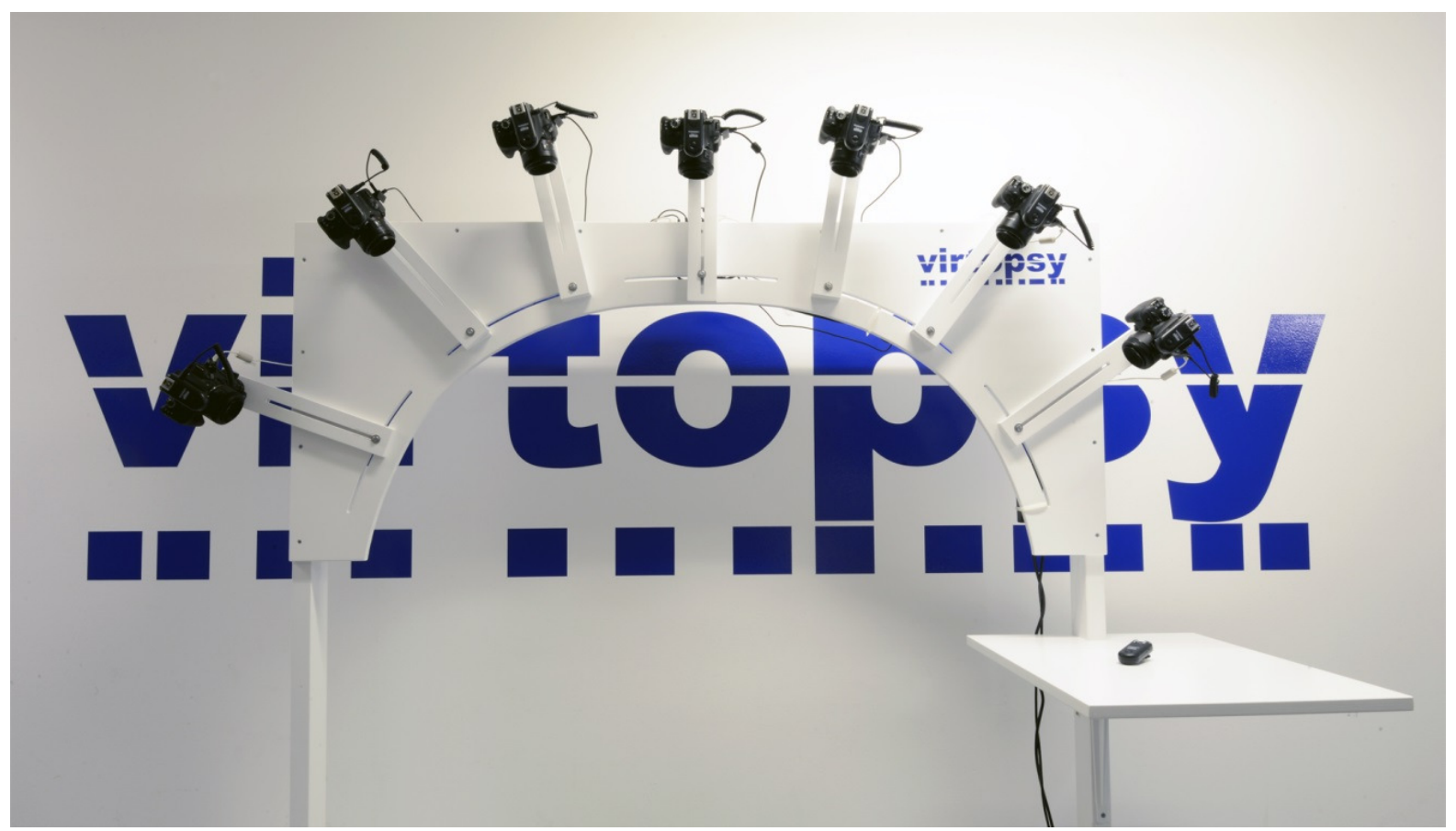

Fig. 1. Setup of the multi-camera rig 


\subsection{Photo-acquisition workflow in combination with PMCT scanning procedures}

In combination with a medical x-ray computed tomography (CT) scanner (Somatom Definition Flash, Siemens Medical Solutions, Forchheim, Germany), the multi-camera rig is used to acquire overlapping multi-view images of a body. For this purpose the multi-camera rig is placed centrally above the CT table in front of the CT gantry (Fig. 2a). With the help of the operating software of the CT scanner (Syngo CT 2012B, VA44A, Siemens Medical Solutions, Forchheim, Germany), the CT table movement is synchronized with the remote shutter release of the multi-camera rig. This enables the CT table to move automatically during the scanning process step-by-step beneath the rig. The step-by-step movement of the CT table is paused every $10 \mathrm{~cm}$ and is initiated by an announcement to manually trigger the remote shutter release (Fig. 2b). This procedure is continued until all photos covering the full length of the body are acquired.

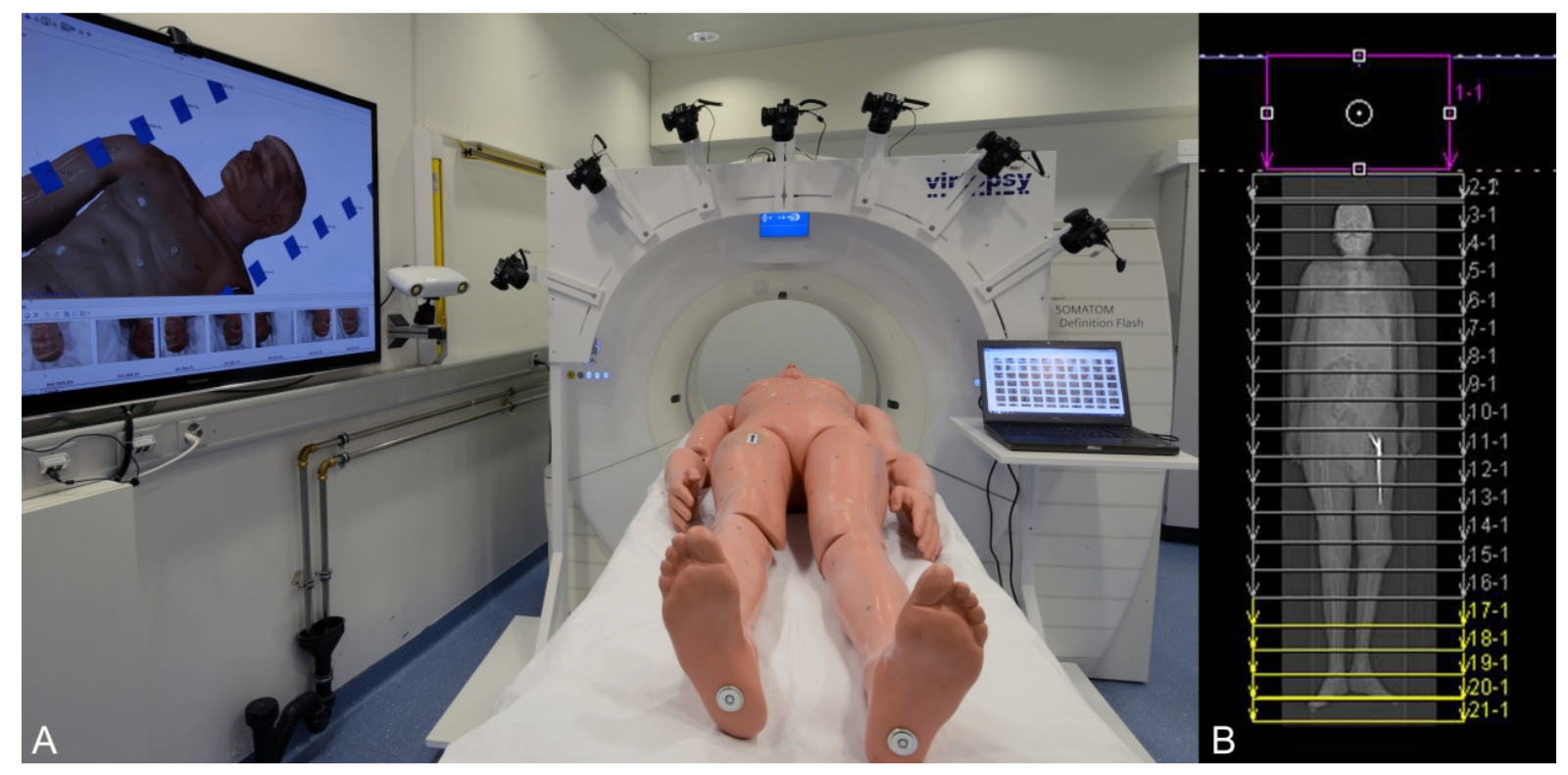

Fig. 2. a: Setup in combination with a medical CT scanner; b: PMCT scout view with a visualization of the modified step-by-step PMCT scanning protocol

\subsection{Photo-acquisition workflow in combination with examination couches, lifting carts and autopsy tables}

Close-range photogrammetry with the help of the multi-camera rig can also be performed manually without a CT scanning setup. In this scenario, the multi-camera rig can be used in combination with a body-carrying setup, such as an examination couch, a lifting cart (Fig. 3a) or an autopsy table (Fig. 3b). The multi-camera rig is placed at the top or bottom end of the body carrying setup and is aligned centrally above the body. Subsequently, the multi-camera rig is moved manually step-by-step across the body-carrying area. Every $10 \mathrm{~cm}$, the rig is stopped, and the remote shutter release is triggered manually by the radio controller. This procedure is continued until the rig is moved to the other end of the body-carrying setup. The use of the multi-camera rig in conjunction with a lifting cart and an autopsy table is shown in Figure 3. 

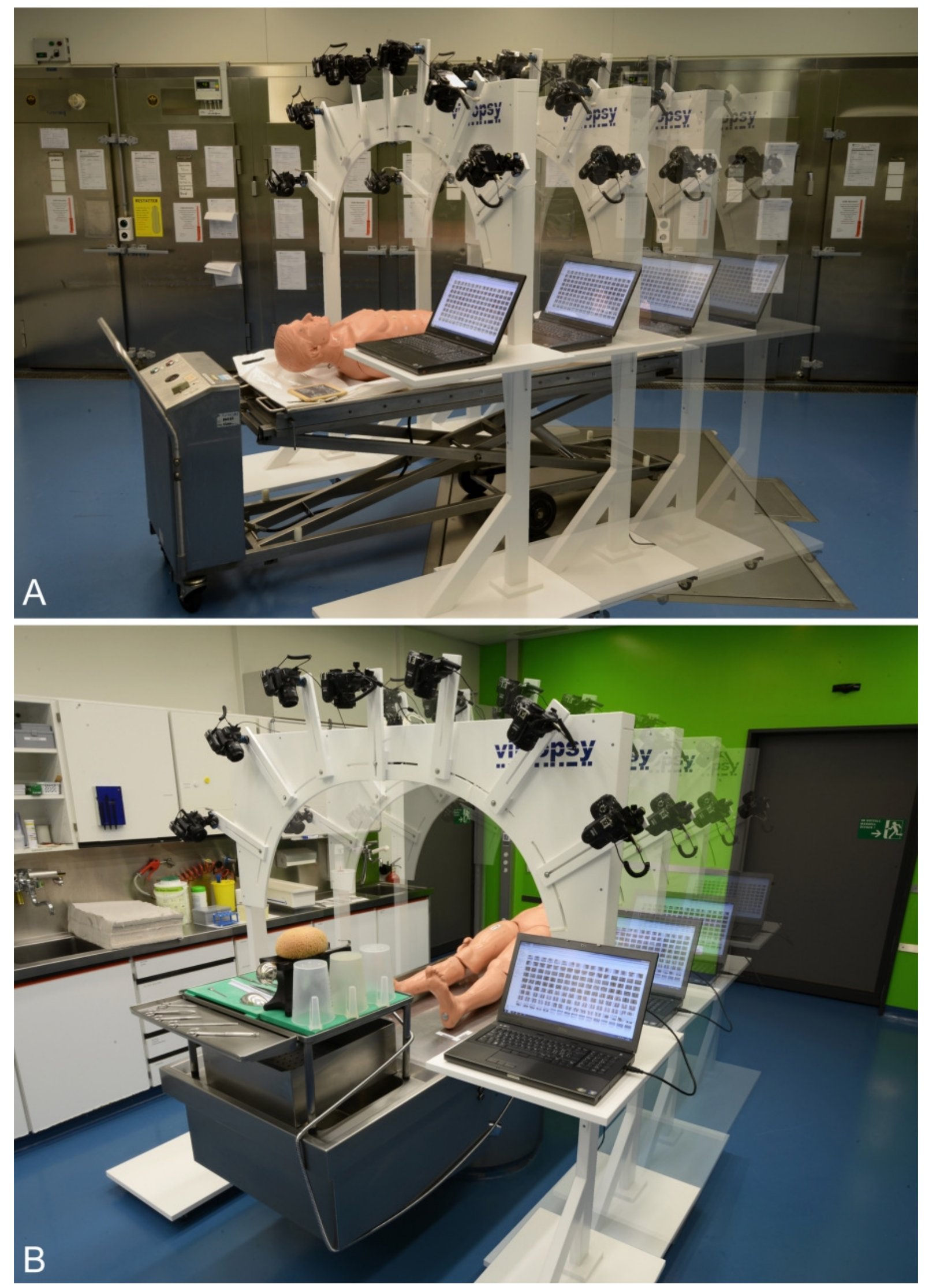

Fig. 3. Use of the multi-camera setup a: in combination with lifting carts; b: in conjunction with autopsy tables 


\subsection{Photogrammetric reconstruction from multi-view images}

Subsequent to the photo acquisition procedure all photos that are collected with the help of the multi-camera rig are transferred manually to a separate workstation (2 Intel $\AA$ Xeon $\AA$ CPUs with 16 cores, Intel Corporation, Santa Clara, USA, 36 GB RAM, Nvidia Quadro® FX 1800 GPU, NVIDIA Corporation, Santa Clara, USA, Microsoft Windows 7, 64 Bit, Service Pack 1 operating system, Microsoft Corporation, Redmond, USA). With the help of PhotoScan (Agisoft PhotoScan Professional Edition, Version 1.2.4, Agisoft LLC, St. Petersburg, Russia), the photogrammetric reconstruction is computed. All seven cameras on the multi-camera rig are used uncalibrated and the default parameters in the PhotoScan Software are used for the computation procedure instead. After a textured 3D model is computed, the model is scaled on the basis of the reference markers that are applied to the body's surface prior to the photo acquisition procedure.

\section{Results}

Photo acquisition in conjunction with the automated PMCT scanning procedures lasted 3:34 minutes to acquire 126 overlapping photographs. Photogrammetric reconstructions in PhotoScan delivered a textured 3D model based on a low-resolution mesh in 10:55 minutes. The data size of the 3D model accounted for $6613 \mathrm{KiB}$ and comprised 135430 faces. An exemplary illustration of the computed 3D model is shown in Figure 4.

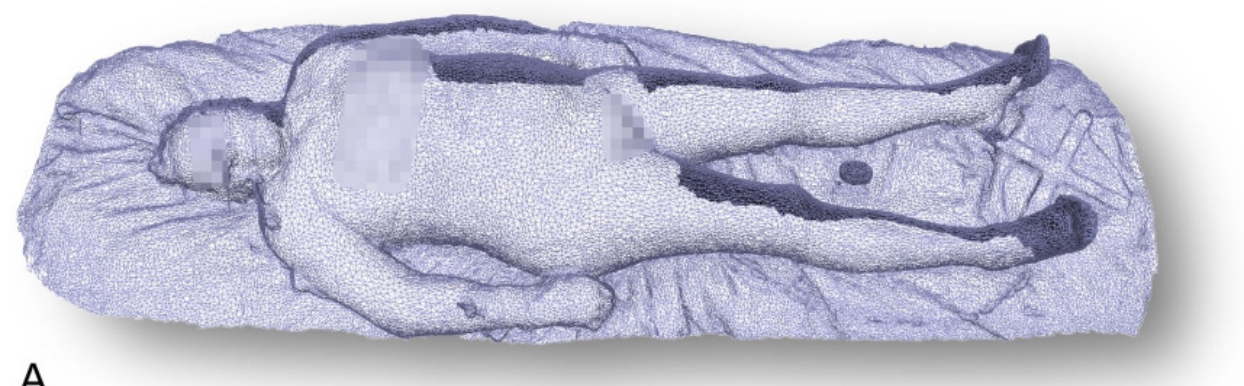

A
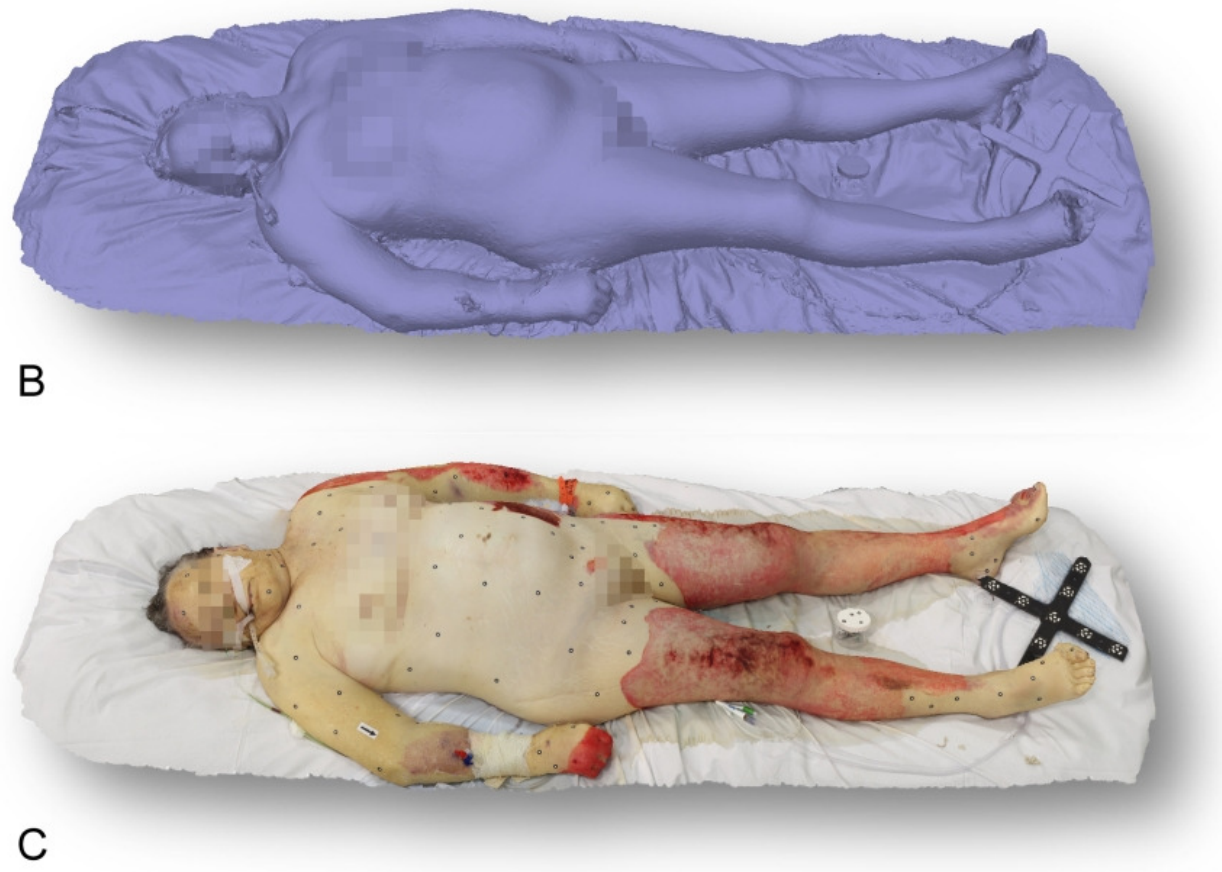

Fig. 4. 3D model based on 126 overlapping photographs a: model displayed in wireframe mode b: model displayed in solid mode c: model displayed in textured mode 


\section{Conclusions}

Based on our preliminary results, the mobile, multi-camera rig offers short scanning and computation times for 3D full-body documentation. The setup can help standardize 3D full-body scanning procedures and can be used to document the three-dimensional surface of deceased and living people. In comparison to other commercially available 3D scanning systems, the multi-camera rig offers a great advantage in terms of its low acquisition and maintenance costs. Overall, the multi-camera setup appears to be a practical and fast 3D full-body scanning alternative for forensic investigations. However, further studies with a larger sample size are necessary to evaluate the feasibility of the system.

\section{Acknowledgements}

The authors express their gratitude to Emma Louise Kessler, M.D. for her generous donation to the Zurich Institute of Forensic Medicine, University of Zurich, Switzerland.

\section{Disclosure}

The authors declare that there are no conflicts of interest.

\section{References}

[1] W. Brüschweiler, M. Braun, R. Dirnhofer, und M. J. Thali, „Analysis of patterned injuries and injury-causing instruments with forensic 3D/CAD supported photogrammetry (FPHG): an instruction manual for the documentation process“, Forensic Sci. Int., Bd. 132, Nr. 2, S. 130-138, März 2003.

[2] M. J. Thali, M. Braun, und R. Dirnhofer, „Optical 3D surface digitizing in forensic medicine: 3D documentation of skin and bone injuries", Forensic Sci. Int., Bd. 137, Nr. 2-3, S. 203-208, Nov. 2003.

[3] M. J. Thali, M. Braun, W. Brueschweiler, und R. Dirnhofer, „'Morphological imprint': determination of the injury-causing weapon from the wound morphology using forensic 3D/CAD-supported photogrammetry“, Forensic Sci. Int., Bd. 132, Nr. 3, S. 177-181, Apr. 2003.

[4] M. J. Thali, M. Braun, T. H. Markwalder, W. Brueschweiler, U. Zollinger, N. J. Malik, K. Yen, und R. Dirnhofer, „Bite mark documentation and analysis: the forensic 3D/CAD supported photogrammetry approach“, Forensic Sci. Int., Bd. 135, Nr. 2, S. 115-121, Aug. 2003.

[5] M. J. Thali, M. Braun, U. Buck, E. Aghayev, C. Jackowski, P. Vock, M. Sonnenschein, und R. Dirnhofer, „VIRTOPSY - scientific documentation, reconstruction and animation in forensic: individual and real 3D data based geo-metric approach including optical body/object surface and radiological CT/MRI scanning", J Forensic Sci, Bd. 50, Nr. 2, S. 428-42, 2005.

[6] U. Buck, S. Naether, M. Braun, S. Bolliger, H. Friederich, C. Jackowski, E. Aghayev, A. Christe, P. Vock, R. Dirnhofer, und M. J. Thali, „Application of 3D documentation and geometric reconstruction methods in traffic accident analysis: With high resolution surface scanning, radiological MSCT/MRI scanning and real data based animation“, Forensic Sci. Int., Bd. 170, Nr. 1, S. 20-28, Juli 2007.

[7] U. Buck, S. Naether, B. Räss, C. Jackowski, und M. J. Thali, „Accident or homicide - Virtual crime scene reconstruction using 3D methods“, Forensic Sci. Int., Bd. 225, Nr. 1-3, S. 75-84, Feb. 2013.

[8] J. Tschui, N. Feddern, N. Schwendener, L. Campana, S. Utz, M. Schweizer, C. Jackowski, und W. D. Zech, „When the prey gets too big: an uncommon road accident involving a motorcyclist, a car and a bird", Int. J. Legal Med., Bd. 130, Nr. 2, S. 463-467, Apr. 2015.

[9] A. Persson, M. Lindblom, und C. Jackowski, „A state-of-the-art pipeline for postmortem CT and MRI visualization: from data acquisition to interactive image interpretation at autopsy", Acta Radiol., Bd. 52, Nr. 5, S. 522-536, Jan. 2011.

[10] C. Lundström, A. Persson, S. Ross, P. Ljung, S. Lindholm, F. Gyllensvärd, und A. Ynnerman, "State-of-the-art of visualization in post-mortem imaging", APMIS, Bd. 120, Nr. 4, S. 316-326, Apr. 2012. 
[11] S. E. Westphal, J. Apitzsch, T. Penzkofer, A. H. Mahnken, und R. Knüchel, „Virtual CT autopsy in clinical pathology: feasibility in clinical autopsies“, Virchows Arch., Bd. 461, Nr. 2, S. 211-219, Juni 2012.

[12] D. Wichmann, F. Obbelode, H. Vogel, W. W. Hoepker, A. Nierhaus, S. Braune, G. Sauter, K. Pueschel, und S. Kluge, „Virtual Autopsy as an Alternative to Traditional Medical Autopsy in the Intensive Care UnitA Prospective Cohort Study", Ann. Intern. Med., Bd. 156, Nr. 2, S. 123-130, Jan. 2012.

[13] M. Baglivo, S. Winklhofer, G. M. Hatch, G. Ampanozi, M. J. Thali, und T. D. Ruder, „The rise of forensic and post-mortem radiology-Analysis of the literature between the year 2000 and 2011", J. Forensic Radiol. Imaging, Bd. 1, Nr. 1, S. 3-9, Jan. 2013.

[14] P. M. Flach, D. Gascho, W. Schweitzer, T. D. Ruder, N. Berger, S. G. Ross, M. J. Thali, und G. Ampanozi, „Imaging in forensic radiology: an illustrated guide for postmortem computed tomography technique and protocols", Forensic Sci. Med. Pathol., Bd. 10, Nr. 4, S. 583-606, Apr. 2014.

[15] T. D. Ruder, M. J. Thali, und G. M. Hatch, „Essentials of forensic post-mortem MR imaging in adults“, Br. J. Radiol., Bd. 87, Nr. 1036, Apr. 2014.

[16] W. Brüschweiler, M. Braun, H. J. Fuchser, und R. Dirnhofer, „Photogrammetrische Auswertung von Haut- und Weichteilwunden sowie Knochenverletzungen zur Bestimmung des Tatwerkzeuges - grundlegende Aspekte“, Rechtsmedizin, Bd. 7, Nr. 3, S. 76-83.

[17] J. Subke, H.-D. Wehner, F. Wehner, und S. Szczepaniak, „Streifenlichttopometrie (SLT): A new method for the three-dimensional photorealistic forensic documentation in colour", Forensic Sci. Int., Bd. 113, Nr. 1-3, S. 289-295, Sep. 2000.

[18] M. J. Thali, M. Braun, W. Brüschweiler, und R. Dirnhofer, „Matching tire tracks on the head using forensic photogrammetry“, Forensic Sci. Int., Bd. 113, Nr. 1-3, S. 281-287, Sep. 2000.

[19] G. Sansoni, C. Cattaneo, M. Trebeschi, D. Gibelli, D. Porta, und M. Picozzi, „Feasibility of Contactless 3D Optical Measurement for the Analysis of Bone and Soft Tissue Lesions: New Technologies and Perspectives in Forensic Sciences", J. Forensic Sci., Bd. 54, Nr. 3, S. 540-545, Mai 2009.

[20] L. C. Ebert, W. Ptacek, S. Naether, M. Fürst, S. Ross, U. Buck, S. Weber, und M. Thali, „Virtobot-a multi-functional robotic system for 3D surface scanning and automatic post mortem biopsy“, Int. J. Med. Robot., Bd. 6, Nr. 1, S. 18-27, März 2010.

[21] W. Schweitzer, E. Röhrich, M. Schaepman, M. J. Thali, und L. Ebert, „Aspects of 3D surface scanner performance for post-mortem skin documentation in forensic medicine using rigid benchmark objects“, J. Forensic Radiol. Imaging, Bd. 1, Nr. 4, S. 167-175, Okt. 2013.

[22] L. C. Ebert, W. Ptacek, R. Breitbeck, M. Fürst, G. Kronreif, R. M. Martinez, M. Thali, und P. M. Flach, „Virtobot 2.0: the future of automated surface documentation and CT-guided needle placement in forensic medicine“, Forensic Sci. Med. Pathol., Bd. 10, Nr. 2, S. 179-186, Jan. 2014.

[23] L. Campana, R. Breitbeck, R. Bauer-Kreuz, und U. Buck, „3D documentation and visualization of external injury findings by integration of simple photography in CT/MRI data sets (IprojeCT)“, Int. J. Legal Med., Bd. 130, Nr. 3, S. 787-797, Okt. 2015.

[24] P. Urbanová, P. Hejna, und M. Jurda, „Testing photogrammetry-based techniques for three-dimensional surface documentation in forensic pathology", Forensic Sci. Int., Bd. 250, S. 77-86, Mai 2015.

[25] K. M. Robinette, H. Daanen, und E. Paquet, „The CAESAR project: a 3-D surface anthropometry survey", in Second International Conference on 3-D Digital Imaging and Modeling, 1999. Proceedings, 1999, S. 380-386.

[26] B. Allen, B. Curless, und Z. Popović, „The Space of Human Body Shapes: Reconstruction and Parameterization from Range Scans", in ACM SIGGRAPH 2003 Papers, New York, NY, USA, 2003, S. 587-594.

[27] P. Treleaven, „Sizing us up“, IEEE Spectr., Bd. 41, Nr. 4, S. 28-31, Apr. 2004.

[28] J. Tong, J. Zhou, L. Liu, Z. Pan, und H. Yan, „Scanning 3D Full Human Bodies Using Kinects“, IEEE Trans. Vis. Comput. Graph., Bd. 18, Nr. 4, S. 643-650, Apr. 2012.

[29] J. I. Echevarria, D. Bradley, D. Gutierrez, und T. Beeler, „Capturing and Stylizing Hair for 3D Fabrication“, ACM Trans Graph, Bd. 33, Nr. 4, S. 125:1-125:11, Juli 2014. 
[30] C.-H. J. Tzou, N. M. Artner, I. Pona, A. Hold, E. Placheta, W. G. Kropatsch, und M. Frey, "Comparison of three-dimensional surface-imaging systems", J. Plast. Reconstr. Aesthet. Surg., Bd. 67, Nr. 4, S. 489-497, Apr. 2014.

[31] T. Beeler, „Passive Spatiotemporal Geometry Reconstruction of Human Faces at High Fidelity“, IEEE Comput. Graph. Appl., Bd. 35, Nr. 3, S. 82-90, Mai 2015.

[32] N. D’Apuzzo, „Modeling human faces with multi-image photogrammetry“, in Electronic Imaging 2002, 2002, S. 191-197.

[33] M. Abreu de Souza, S. Robson, und J. C. Hebden, „A Photogrammetric Technique for Acquiring Accurate Head Surfaces of Newborn Infants for Optical Tomography Under Clinical Conditions“, Photogramm. Rec., Bd. 27, Nr. 139, S. 253-271, Sep. 2012.

[34] I. Detchev, M. Mazaheri, S. Rondeel, und A. Habib, „Calibration of multi-camera photogrammetric systems“, ISPRS - Int. Arch. Photogramm. Remote Sens. Spat. Inf. Sci., Bd. XL-1, S. 101-108, Nov. 2014.

[35] M. Pesce, L. M. Galantucci, G. Percoco, und F. Lavecchia, „A Low-cost Multi Camera 3D Scanning System for Quality Measurement of Non-static Subjects“, Procedia CIRP, Bd. 28, S. 88-93, 2015.

[36] M. M. de Sainte Croix, D. Gauld, A. H. Forgie, und R. Lowe, „Three-dimensional imaging of human cutaneous forearm bite marks in human volunteers over a 4 day period“, J. Forensic Leg. Med., Bd. 40, S. 34-39, Mai 2016.

[37] A. Leipner, R. Baumeister, M. J. Thali, M. Braun, E. Dobler, und L. C. Ebert, „Multi-camera system for 3D forensic documentation", Forensic Sci. Int., Bd. 261, S. 123-128, Apr. 2016.

[38] F. Remondino und S. El-Hakim, „Image-based 3D Modelling: A Review“, Photogramm. Rec., Bd. 21, Nr. 115, S. 269-291, Sep. 2006.

[39] F. Remondino, S. F. El-Hakim, A. Gruen, und L. Zhang, „Turning images into 3-D models“, IEEE Signal Process. Mag., Bd. 25, Nr. 4, S. 55-65, Juli 2008. 